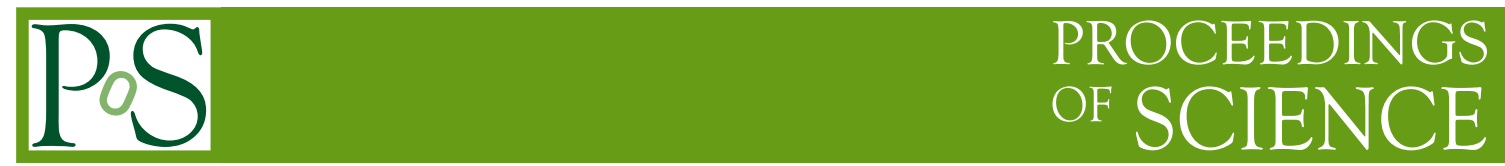

\title{
A walk through the world of chiral dynamics
}

\author{
Ulf-G. Meißner*t \\ Helmholtz-Institut für Strahlen- und Kernphysik and Bethe Center for Theoretical Physics \\ Universität Bonn, D-53115 Bonn, Germany \\ Institute for Advanced Simulation, Institut für Kernphysik and Jülich Center for Hadron Physics, \\ Forschungszentrum Jülich, D-52425 Jülich, Germany \\ E-mail: meissner@hiskp.uni-bonn.de
}

Hadron-hadron scattering lengths are fine probes of our understanding of nonperturbative QCD. I discuss the status of a variety of scattering processes sensitive to the spontaneous and explicit chiral symmetry breaking of QCD, such as pion-pion, pion-kaon, pion-nucleon, antikaon-nucleon and Goldstone boson scattering off $D$-mesons. The fruitful interplay of theory, experiment and lattice QCD is emphasized. I point out what has to be done in these fields to gain further insight into these fundamental parameters.

The 7th International Workshop on Chiral Dynamics,

August 6 -10, 2012

Jefferson Lab, Newport News, Virginia, USA

\footnotetext{
*Speaker.

${ }^{\dagger}$ Work supported in part by DFG, EU and BMBF.
} 


\section{Why hadron-hadron scattering?}

In 1966, Weinberg considered pion scattering off hadrons using current algebra techniques [1]. For pion scattering on a target with mass $m_{t}$ and isospin $T_{t}$, the corresponding scattering lengths for total isospin $T$ read

$$
a_{T}=-\frac{L}{1+M_{\pi} / m_{t}}\left[T(T+1)-T_{t}\left(T_{t}+1\right)-2\right],
$$

with $M_{\pi}$ the charged pion mass. For pion scattering on a pion ["the more complicated case"], he found

$$
a_{0}=\frac{7}{4} L, \quad a_{2}=-\frac{1}{2} L, \quad L=\frac{g_{V}^{2} M_{\pi}}{8 \pi F_{\pi}^{2}} \simeq 0.1 M_{\pi}^{-1},
$$

with $g_{V} \simeq 1$ the vector coupling and $F_{\pi} \simeq 92 \mathrm{MeV}$ the weak pion decay constant. The predictions were on one side amazing due to their extreme simplicity and on the other side surprising, as one believed that scattering lengths should be of the order of $1 \mathrm{fm}$, the typical hadronic length scale - and not much smaller as given by these equations. The physics behind this suppression is well understood - the Goldstone boson nature of the pions requires decoupling from any given field as external momenta go to zero (in the limit of vanishing quark / pion masses). Corrections to these predictions can be worked out consistently in chiral perturbation theory (CHPT) and variants thereof, as will be discussed in the next sections. CHPT is the effective field theory (EFT) of the Standard Model at low energies and allows one to systematically explore the consequences of the spontaneous and explicit chiral symmetry breaking in QCD. Given the aims and scope of this meeting, it is therefore natural to ask: what have we learned since the seminal work of Weinberg? In the following, I will give a very personal answer to this question and hopefully convince the reader that hadron-hadron scattering is a fine tool to gain insight into the strong interactions in the nonperturbative regime.

\section{Chiral symmetry and the essence of chiral perturbation theory}

This section serves as a warm up - as CHPT and extensions thereof will be used to analyze various hadron-hadron scattering processes, a few remarks are in order. As already stated, CHPT explores the consequences of chiral symmetry breaking in QCD. As in any effective field theory, a power counting based on the scale separation lets one organize the string of contributions to a given matrix element or Greens function. The power of chiral symmetry manifests itself in the relations between many processes. A particularly nice example of this are the next-to-leading order lowenergy constants (LECs) of the chiral effective pion-nucleon Lagrangian, commonly denoted as $c_{i}$. These can e.g. be determined from data on low energy pion-nucleon scattering to some precision. They can then be used in the chiral EFT description of the nuclear forces, as they play an important role in the two-pion exchange contributions of the two-nucleon forces and also in the leading longrange part of the three-nucleon force. It is also important to stress that while the number of LECs increases with higher orders, this is not necessarily prolific for basic processes. E.g., elastic $\pi \pi$ scattering to one loop features four LECs, at two loops there are only 2 new LECs, as all other $\mathscr{O}\left(p^{6}\right)$ local operators simply lead to a quark mass renormalizations of the $\mathscr{O}\left(p^{4}\right)$ LECs. Further, the predictions of CHPT can be sharpened by combining it with other methods such as dispersion 


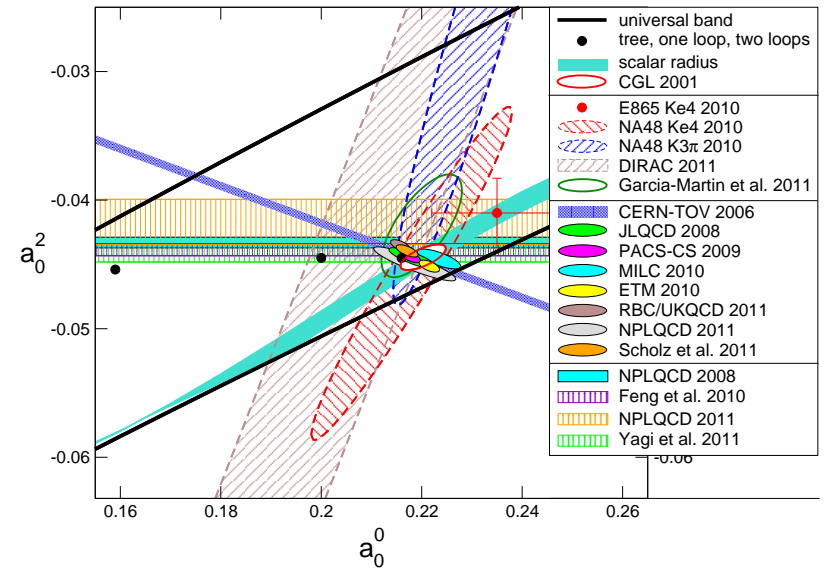

Figure 1: Theoretical predictions for the Swave $\pi \pi$ scattering lengths in comparison to experimental data as well as direct and indirect lattice determinations. Figure courtesy of Heiri Leutwyler [5].

relations, coupled-channel approaches, lattice simulations and so on. As I will show, this can lead to incredibly precise predictions in some cases, but one should be aware that there is no free lunch - as will be discussed in the following.

\section{Lesson 1: Pion-pion scattering}

Elastic pion-pion scattering ( $\pi \pi \rightarrow \pi \pi$ ) is the purest process in two-flavor chiral dynamics as the up and the down quark masses are really small compared to any other hadronic mass scale. At threshold the scattering amplitude is given in terms of two numbers, the scattering lengths $a_{0}$ and $a_{2}$. Most interesting is the history of the prediction for $a_{0}$ : At leading order (LO) (tree graphs in CHPT) one has $a_{0}=0.16$ [1]. The NLO (one-loop) corrections were worked out by Gasser and Leutwyler in 1983, $a_{0}=0.20 \pm 0.01$ [2]. The fairly large correction can be understood in terms of the strong $\pi \pi$ final-state interactions in this partial wave. At NNLO (2-loops), one finds $a_{0}=$ $0.217 \pm 0.009$ [3] and this was considerably sharpened by matching the 2-loop representation to the Roy equation solution, resulting in $a_{0}=0.220 \pm 0.005$ [4]. This is an amazingly precise prediction for a low-energy QCD observable. Incidentally, a similarly accurate prediction was achieved for $a_{2}, a_{2}=-0.0444 \pm 0.0010$, but here the corrections to the LO result, $a_{2}^{\mathrm{LO}}=-0.0042$, are very small due to the very weak $\pi \pi$ interactions in isospin 2 . This visible improvement in accuracy compared to the two-loop predictions was due to the inclusion of data from higher energies in the dispersion relations with better accuracy than this is possible through the finite number of LECs. Also, the two scattering lengths serve as subtraction constants in the Roy equations and are thus much tighter constrained than in the pure chiral expansion.

Given such precise predictions - how about experiment? The analysis of the $\pi \pi$ final-state interactions in $K_{e 4}$ and the cusp in $K^{0} \rightarrow 3 \pi^{0}$ decays has proven to lead to the most precise determinations of the scattering lengths. An alternative it the measurement of lifetime of pionium, but this is experimentally more difficult and thus less accurate. From kaon decays using a particularly tailored non-relativistic EFT [6], one obtains $a_{0}^{0}=0.2210 \pm 0.0047_{\text {stat }} \pm 0.0040_{\text {sys }}$ and $a_{0}^{2}=-0.0429 \pm 0.0044_{\text {stat }} \pm 0.0028_{\text {sys }}$ [7]. The pionium lifetime measurement leads to $\left|a_{0}^{0}-a_{0}^{2}\right|=$ $0.2533_{-0.0078}^{+0.000073}$, where the first/second error is statistical/systematic [8]. The agreement with the prediction from Ref. [4] is stunning. In addition, there are direct and indirect lattice determinations of these fundamental parameters. Here, "direct" refers to using the Lüscher method and 


\begin{tabular}{|l|c|c|c|c|}
\hline & Tree [1,14] & 1-loop [15, 16] & 2-loop [17] & RS [18] \\
\hline$a_{0}^{1 / 2}$ & +0.14 & $+0.18 \pm 0.03$ & $+0.220[0.17 \ldots 0.225]$ & $+0.224 \pm 0.022$ \\
$a_{0}^{3 / 2}$ & -0.07 & $-0.05 \pm 0.02$ & $-0.047[-0.075 \ldots-0.04]$ & $-0.0448 \pm 0.0077$ \\
\hline
\end{tabular}

Table 1: Chiral predictions at LO, NLO and NNLO for the $\pi K$ scattering lengths and from Roy-Steiner (RS) equations. The uncertainty for the 2-loop results is taken from the various parameter variations discussed in [17].

extracting the scattering length from the measured energy shift while "indirect" means that the LECs $\ell_{3}$ and $\ell_{4}$ have been extracted from the pion decay constant and mass whereas $\ell_{1,2}$ have been taken from other sources. The grand picture is presented in Fig. 1 and shows a beautiful consistency. This is truly one of the finest tests of the Standard Model at low energies. However, not all is well - a direct lattice determination of $a_{0}$ is still missing and the lattice practitioners are urged to provide this so important number. Such a calculation is, of course, technically challenging because of the disconnected diagrams, but time is ripe for doing it.

\section{Lesson 2: Pion-kaon scattering}

The purest scattering process in chiral dynamics involving strange quarks is elastic pion-kaon scattering, $\pi K \rightarrow \pi K$. Again, at threshold the scattering amplitude is given in terms of two numbers, namely the scattering lengths $a_{1 / 2}$ and $a_{3 / 2}$. Before discussing the status of the $\pi K$ scattering lengths, I want to address a few mysteries surrounding the $s$ quark. In standard three-flavor CHPT, it is treated like the $u, d$ quarks. However: is the strange quark really light as $m_{s} \sim 100 \mathrm{MeV} \sim \Lambda_{\mathrm{QCD}}$ ? This is reflected in the expansion parameter: $\xi_{s}=M_{K}^{2} /\left(4 \pi F_{\pi}\right)^{2} \simeq 0.18$, which is much bigger than its SU(2) equivalent: $\xi=M_{\pi}^{2} /\left(4 \pi F_{\pi}\right)^{2} \simeq 0.014$. In fact, many predictions of SU(3) CHPT work quite well, but there are also indications of bad convergence in some recent lattice calculations, see e.g. Refs. [9, 10]. A possible solution to this is offered by reordering techniques, see [11]. Also, since many years there have been speculations that the three-flavor condensate $\Sigma(3)$ is very much suppressed compared to its two-flavor cousin $\Sigma(2)$. E.g. Moussallam performed a sum rule study and found a sizeable suppression, $\Sigma(3)=\Sigma(2)[1-0.54 \pm 0.27]$ [12] whereas a recent lattice study finds a more standard relation $\Sigma(3)=\Sigma(2)[1-0.23 \pm 0.39]$ [13]. Note that in both cases the uncertainties are large. The history of the CHPT predictions for the scattering lengths is given in Tab. 1 together with the results of the Roy-Steiner (RS) equations [18]. The agreement with the two-loop predictions (central values) is quite satisfactory, but the precision achieved in the RS framework is not as good as in the case of $\pi \pi$ scattering. This is largely due to the worse and partly inconsistent data base. Similarly, the scattering lengths extracted from these data are not very precise and fairly scattered, see e.g. Fig. 1 in Ref. [15]. More recent measurements in D- and B-meson decays with high statistics $[19,20,21,22]$ allow in principle for a better determination of $a_{1 / 2}$ and $a_{3 / 2}$, but none of these has performed an isospin decomposition. This is urgently called for. In principle, lattice QCD can have some impact here. However, the present situation as summarized for $a_{1 / 2}$ in Fig. 2 (the date are taken from the recent paper [23], see also the references therein) is everything but satisfactory, there is a large spread of the results. For $a_{3 / 2}$, there is better agreement between the lattice results but also some tension with the value obtained from the RS analysis. Clearly, more work is needed to clear up the situation for this process. 


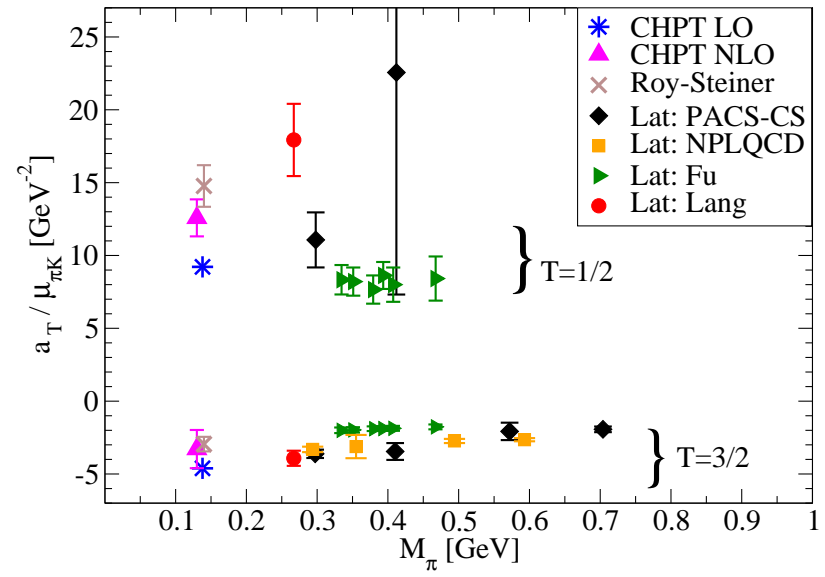

Figure 2: The S-wave $\pi K$ scattering lengths from the lattice in comparison to $\mathrm{LO}$ and NLO CHPT and Roy-Steiner determinations. Data as collected in Ref. [23]. For better comparison, the scattering lengths are normalized to the reduced mass $\mu_{\pi K}$ of the pion-kaon system.

\section{Lesson 3: Pion-nucleon scattering}

Let me now turn to elastic pion-nucleon scattering, $\pi N \rightarrow \pi N$, which is the simplest (and also the most fundamental) scattering process involving nucleons. As in the case of pion-kaon scattering, one has total isospin $1 / 2$ and $3 / 2$. Often used are the isoscalar and isovector scattering length, $a^{+}$and $a^{-}$, respectively, with $a_{3 / 2}=a^{+}-a^{-}$and $a_{1 / 2}=a^{+}+2 a^{-}$. The LO prediction for the isoscalar/isovector scattering length is quite intriguing:

$$
a_{\mathrm{LO}}^{+}=0, a_{\mathrm{LO}}^{-}=\frac{1}{1+M_{\pi} / m_{p}} \frac{M_{\pi}^{2}}{8 \pi F_{\pi}^{2}}=79.5 \cdot 10^{-3} / M_{\pi} .
$$

Much is known about the chiral corrections to these results that were first addressed in [24]. While the chiral expansion for $a^{-}$converges fast [25], there are large cancellations in $a^{+}$, so that even its sign is not known from scattering data, see e.g. table 3 in [26] (see also the more recent work in Ref. [27]). However, there is a wonderful alternative to get a hand on these scattering lengths, namely hadronic atoms. These are electromagnetic bound states of two oppositely charged hadrons. Due to the large spatial extent of these objects, the strong interactions lead to small perturbations in the observed level spectrum. In particular, there is a shift of the ground state energy $\left(\Delta E_{1 s}\right)$ and further, due to channel coupling, this level acquires a width $\Gamma_{1 s}$. Due to the small three-momenta in such a system, we are dealing essentially with scattering at zero energy or, stated differently, the energy shift and width can be expressed in terms of the corresponding scattering lengths. There are many species of such hadronic atoms, like pionium $\left(\pi^{+} \pi^{-}\right)$already discussed, pionic hydrogen $\left(\pi^{-} p\right)$, pionic deuterium $\left(\pi^{-} d\right)$ and their kaonic cousins $\left(K^{-} p, K^{-} d\right)$. Hadronic atoms can be analyzed most systematically and precisely in suitably tailored non-relativistic EFTs, see [28] for a comprehensive review. For the case of pion-nucleon scattering, the corresponding high-precision theoretical framework for pionic hydrogen and pionic deuterium has been provided in Refs. [29] and [30], respectively (see also the contribution from Martin Hoferichter to these proceedings). On the experimental side, superb experiments have been performed at PSI for many years, culminating in precise measurements of the energy shift and width of pionic hydrogen [31] and the energy shift in pionic deuterium [32]. The analysis of these data within these EFTs leads to a very precise extaction of the scattering lengths [30]

$$
a^{+}=(7.6 \pm 3.1) \cdot 10^{-3} / M_{\pi}, \quad a^{-}=(86.1 \pm 0.9) \cdot 10^{-3} / M_{\pi} .
$$




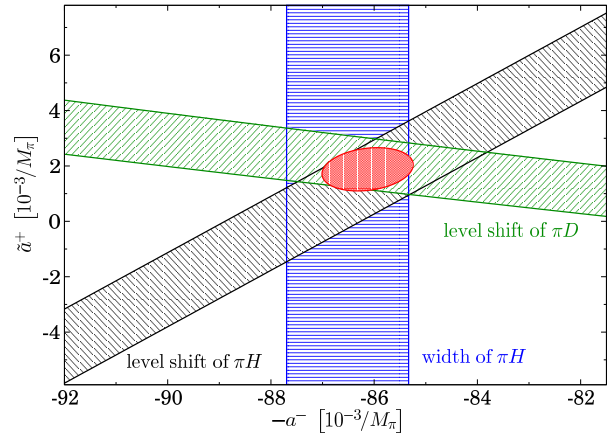

Figure 3: Extraction of the isoscalar and isovector scattering lengths from pionic hydrogen and deuterium. Here, $\tilde{a}^{+}=a^{+}+\frac{1}{1+M_{\pi} / m_{p}} \times$ $\left\{\frac{\Delta M_{\pi}^{2}}{\pi F_{\pi}} c_{1}-2 \alpha f_{1}\right\}$, with $\Delta M_{\pi}^{2}=M_{\pi}^{2}-M_{\pi^{0}}^{2}$, $\alpha$ the fine structure constant and $c_{1} / f_{1}$ are strong/electromagnetitic NLO LECs with $c_{1} \simeq$ $0.9 \mathrm{GeV}^{-1}$ and $\left|f_{1}\right| \leq 1.4 \mathrm{GeV}^{-1}$. Figure courtesy of Martin Hoferichter.

Note that the value for the isovector scattering length differs by just $8 \%$ form the $\mathrm{LO}$ value, whereas the isocalar one is positive and only slightly larger in value than various contributions to it. This underlines that only within a consistent framework like the employed EFT one is able to extract this value. Also, using the GMO sum rule, the authors of [30] find a precise value for the pionnucleon coupling constant, $g_{\pi N}^{2} /(4 \pi)=13.69(12)(15)$, where the first error stems from the scattering lengths and the second one from the integral over the $\pi^{-} p$ cross section. This is consistent with other determinations from pion-nucleon scattering or from peripheral nucleon-nucleon phase shifts.

\section{Lesson 4: Antikaon-nucleon scattering}

Next, I consider the reaction $K^{-} p \rightarrow K^{-} p$. It is a fundamental scattering process with strange quarks involving baryons. The dynamics of this process is driven by channel couplings and leads to the dynamic generation of the $\Lambda(1405)$ resonance $[33,34]$ that resides between the $\pi \Sigma$ and $\bar{K} N$ thresholds and is certainly not a simple three-quark state. This reaction is therefore a major playground of unitarized CHPT (UCHPT). Due to the open channels below the $K^{-} p$ threshold, the two scattering lengths $a_{0}$ and $a_{1}$ are complex-valued quantities, which means that in this case we deal with four numbers. Before continuing, it is important to point out the differences between CHPT and unitarized versions thereof. CHPT is an exact representation of the chiral Greens function of QCD, which expands matrix elements in powers of small momenta and quark masses. Crossing and analyticity are in general fulfilled (if a proper regularization scheme is employed), whereas due to the underlying power counting, unitarity is fulfilled perturbatively. CHPT is formulated in terms of the lowest-lying hadronic degrees of freedom and all effects from resonances are subsumed in the low-energy constants. If one wants to describe resonances explicitely - as it is the case here - some resummation scheme that fulfills 2-body unitarity exactly is needed. There are various formalism available to achieve that, mostly based on Lippman-Schwinger or Bethe-Salpeter equations. In general, one gives up crossing symmetry and also some model-dependence is induced as there is a cornucopia of unitarization schemes. Further, the power counting is not applied to the reaction amplitude but rather to the kernel of the scattering equation, as it is e.g. frequently and successfully done in chiral nuclear effective field theory. Having said that, it is also important to stress that UCHPT has been quite successful in describing various scattering processes and the dynamic generations of resonances like the $\Lambda(1405), S_{11}(1535), S_{11}(1650)$, and others. 

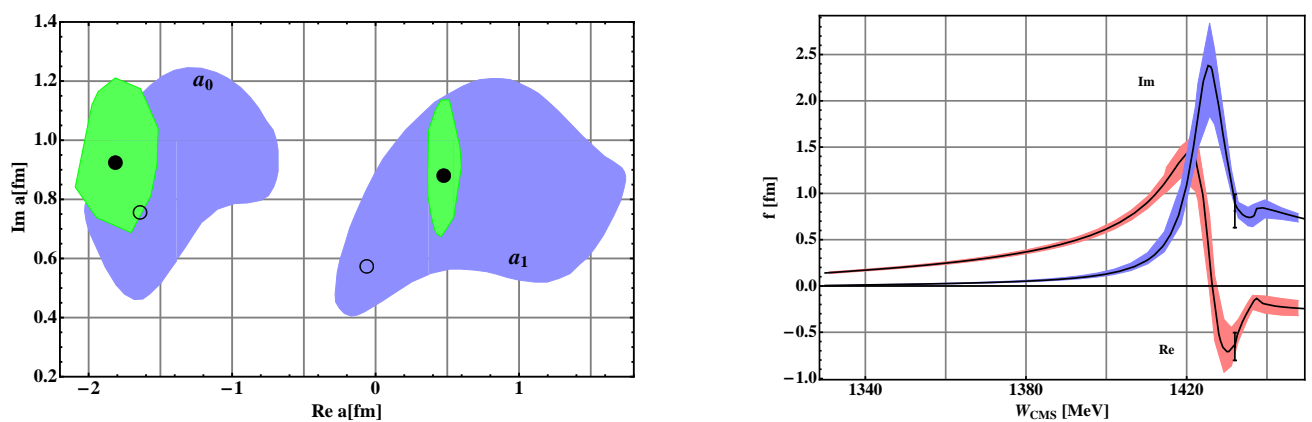

Figure 4: Left panel: Real and imaginary part of the isospin $T=0$ and $T=1 K N \rightarrow K N$ scattering lengths. The light shaded (green) areas correspond to the $1 \sigma$ region of [39] around the central value (full circles). The darker (blue) areas correspond to the $1 \sigma$ region around central value (empty circle) from Ref. [42]. Right panel: Real and imaginary part of the $K^{-} p \rightarrow K^{-} p$ scattering amplitude. The shaded band indicates the uncertainty of the calculation. The data point at $W_{\mathrm{cms}}=M_{K}+m_{p}$ is determined from the energy shift and width of kaonic hydrogen from the SIDDHARTA experiment.

But back to $K^{-} p$ scattering. A long-standing puzzle in these field, namely the inconsistency of the DEAR kaonic hydrogen data with the scattering data (first pointed out in [35] and then confirmed by many others) was recently resolved by the fine experiment of the SIDDHARTA collaboration, who measured the properties of kaonic hydrogen with high precision, $\Delta E_{1 s}=-283 \pm$ 36 (stat) \pm 6 (syst) eV and $\Gamma_{1 s}=541 \pm 89$ (stat) \pm 22 (syst) eV [36]. Based on this, the kaonic hydrogen and the older scattering data can now be analyzed consistently, using the chiral Lagrangian at NLO, as shown by the three groups [37, 38, 39, 40] (see also [41]). Here, I report the results from Ref. [39]. 14 LECs and 3 subtraction constants were fitted to scattering data in the channels $K^{-} p \rightarrow K^{-} p, \bar{K}^{0} n, \Sigma^{ \pm} \pi^{\mp}$, and $\Sigma^{0} \pi^{0}$ for laboratory momenta $p_{\text {lab }} \leq 300 \mathrm{MeV}$ together with the SIDDHARTA data. This allows for good description of the antikaon-proton cross section data and an accurate determination of the scattering lengths,

$$
a_{0}=-1.81_{-0.28}^{+0.30}+i 0.92_{-0.23}^{+0.29} \mathrm{fm}, \quad a_{1}=+0.48_{-0.11}^{+0.12}+i 0.87_{-0.20}^{+0.26} \mathrm{fm} .
$$

The improvement as compared to using scattering data only [42] is clearly visible in Fig. 4 (left panel). These numbers are similar to the ones reported by Ikeda et al. [37, 38]. Therefore, these fundamental chiral SU(3) parameters have now been determined with about an accuracy of $\sim 15 \%$. One can extrapolate the amplitudes of elastic $K^{-} p$ scattering to the subthreshold region, i.e. centerof-mass energies $1330 \leq W_{\mathrm{cms}} \leq 1450 \mathrm{MeV}$. The result is presented in the right panel of Fig. 4 . For both real and imaginary parts of the amplitude the maximum lies close to the $K N$ threshold and is quite narrow, which indicates the presence of a close-by pole. It is also worth mentioning that the error band gets smaller for lower energies, different to the recent analysis by Ikeda et al. [37, 38]. We note that although Ikeda et al. and [39] describe the scattering and bound state data equally well, the subthreshold amplitude is very different. This is presumably due to the different approximation made in these two approaches. Therefore, a truly model-independent determination of this subthreshold amplitude is not yet available. 


\section{Lesson 5: Goldstone boson scattering off $D$ and $D^{\star}$-mesons}

Finally, let us consider scattering the Goldstone boson octet $(\pi, K, \eta)$ off the $D$-meson triplet $\left(D^{0}, D^{+}, D_{s}^{+}\right)$. This involves the positive-parity scalar charm-strange meson $D_{s 0}^{*}(2317)$ which has a very narrow, isospin-violating width and is interpreted by some groups as a molecular $D K$ state. Here, we are mostly interested in the scattering length in the channel with $(S, I)=(1,0)$, as its value can tell us something about the possible nature of the scalar meson. As I will show, this field enjoys a healthy interplay of lattice QCD and UCHPT. But a few general remarks on the calculation of the scattering process $\phi D \rightarrow \phi D$ are in order. This is an interesting problem, as it involves a variety of scales and is also multi-faceted. First, light particles related to the chiral symmetry of QCD are involved, thus we can perform a chiral expansion in momenta and quark masses. Second, as the $D$-meson contain charm quarks, we can exploit heavy quark symmetry and perform an expansion in $1 / m_{c}$. Third, one must consistently include isospin-violation effects. These are on one hand generated by the strong interactions $\left(m_{d} \neq m_{u}\right)$ and on the other hand of electromagnetic origin $\left(q_{u} \neq q_{d}\right)$. In total, one has to consider 16 channels with different total strangeness and isospin. Some of these are perturbative, but most are non-perturbative and require resummation, which can lead to the dynamical generation of molecules.

Before addressing the actual calculations, let me discuss the relation between the scattering length $a$ and the nature of the state under consideration, see Refs. [43, 44] for a derivation:

$$
a=-2\left(\frac{1-Z}{2-Z}\right) \frac{1}{\sqrt{2 \mu \varepsilon}}(1+\mathscr{O}(\sqrt{2 \mu \varepsilon} / \beta))
$$

where $\mu$ and $\varepsilon$ are the reduced mass of the two-hadron system and the binding energy, respectively, and $Z$ is the wave function renormalization constant $(0 \leq Z \leq 1)$. Corrections of the above equation come from neglecting the range of forces, $1 / \beta$, which contains information of the $D_{s} \eta$ channel. Were the $D_{s 0}^{*}(2317)$ a pure $D K$ bound state $(Z=0)$, the value of the $D K(I=0)$ scattering length would be $a=-1.05 \mathrm{fm}$. In [45], new lattice data using the MILC plus Fermilab actions for the channels $D \bar{K}(-1,1),(-1,0), D_{s} K(2,1 / 2), D \pi(0,3 / 2)$ and $D \pi(1,1)$ were analyzed based on the UCHPT formalism developed in [46] (for related work, see [47, 48, 49, 50]). These are more data than previously available allowing in particular for the inclusion of $N_{c}$-suppressed operators of the NLO effective Lagrangian (there are 5 LECs at this order from which 3 are formally subleading in $1 / N_{c}$ and one subtraction constant). If one requires the $D_{s 0}^{*}(2317)$ to be a $D K$-molecule by a proper choice of the subtraction constant (i.e. having a pole at the proper mass in this channel), one has 5 fit parameters that describe the lattice data well, see Fig. 5. In this fit, all parameters come out of natural size and the large- $N_{c}$ hierarchy obeyed. The scattering length in the $D K(I=0)$ channel comes out as $a(D K(I=0))=-0.85_{-0.05}^{+0.07} \mathrm{fm}$, which is consistent with the molecular interpretation (for a more detailed discussion, also concerning a different fit procedure, see [45]). Having pinned down all the LECs, one finds an improved prediction for the isospin-violating width $\Gamma\left(D_{s 0}^{*}(2317)^{+} \rightarrow D_{s}^{+} \pi^{0}\right)=(89 \pm 27) \mathrm{keV}$ (for earlier work in the molecular picture, see [51, 52, 53]). This is very different from typical quark model predictions, which find this width to be about a few $\mathrm{keV}$ [54], and thus an accurate measurement of this quantity is called for. I wish to end with a few remarks on the $D_{s 1}(2460)$. As $M_{D_{s 1}(2460)}-M_{D_{s 0}^{*}(2317)} \simeq M_{D^{*}}-M_{D}$, it is most likely a $D^{\star} K$ molecule (if the $D_{s 0}^{\star}(2317)$ is a $D K$ molecule). Goldstone boson scattering off $D$ - 

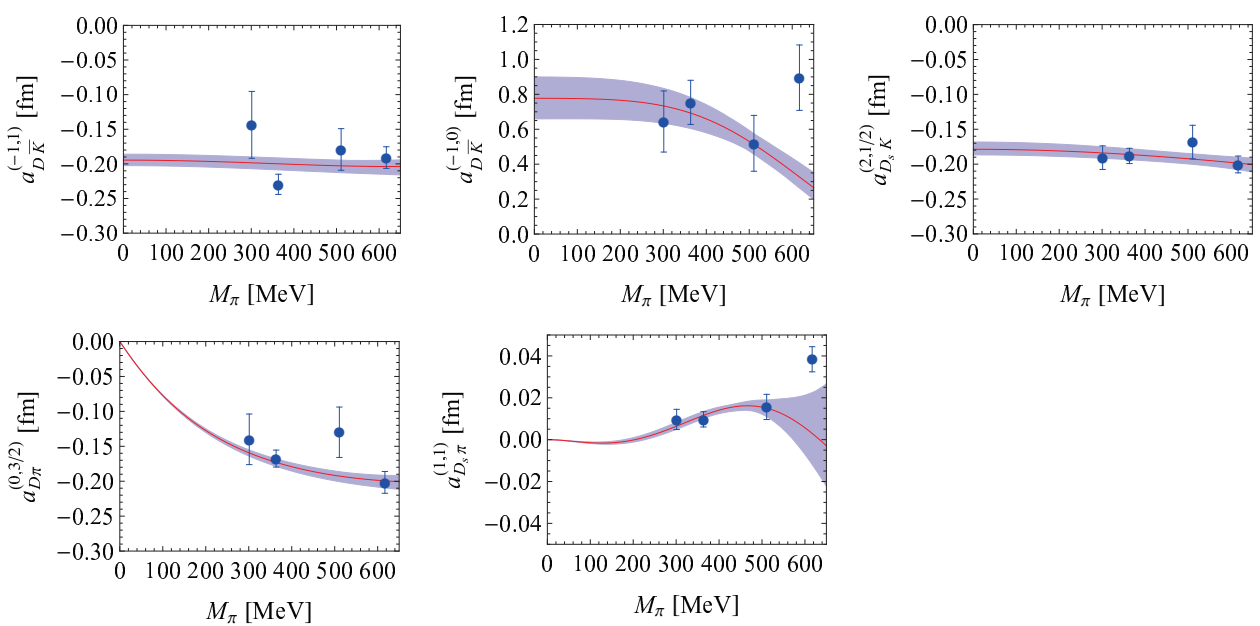

Figure 5: Fit to lattice data for various channels with 5 parameters. The subtraction constant is determined from fixing the pole in the $(S, I)=(1,0)$ channel to $2317.8 \mathrm{MeV}$. The points at the highest pion mass in each channel are not fitted.

and $D^{\star}$-mesons was considered in [49]. The most interesting observation made concerns the mass and binding energy of a molecular state of a heavy meson $(H)$ and a kaon. As its mass is given by $M_{\text {mol }}=M_{K}+M_{H}-\varepsilon$, the mass should depend linearly on the kaon mass, very different from a genuine multi-quark state, whose mass depends linearly on the strange quark and thus quadratically on the kaon mass. This behaviour can, of course, be investigated on the lattice.

\section{Short summary \& outlook}

As I have shown, there has been much progress in our understanding of hadron-hadron scattering lengths since Weinberg's seminal paper in 1966. Most advanced is theory and experiment in the case of pion-pion scattering. Here, lattice QCD still has to provide an acccurate number for $a_{0}$. Matters are much less satisfactory for pion-kaon scattering - as both experiment and lattice QCD have to deliver more precise values for both scattering lengths. A measurement of the properties of $\pi K$ atoms would certainly be very welcome $[55,56]$. Very different to that, the combination of chiral EFTs with precision data has led to a high precision determination of the pion-nucleon scattering lengths $a^{+}$and $a^{-}$. This should challenge lattice practioners to provide a similarly accurate $a b$ initio calculation. In case of antikaon-nucleon scattering, there has been recent progress by analysing scattering data and kaonic hydrogen data provided by SIDDHARTA and the corresponding scattering lengths are now known with an accuracy of about 15\%. Here, a measurement of kaonic deuterium would provide further stringent constraints [57, 58, 59]. Finally, including charm quarks, the interplay of unitarized CHPT and lattice QCD complemented by experiment can deepen our understanding of heavy-light systems, thus providing a bridge from chiral dynamics to the physics of heavy quark flavors.

\section{Acknowledgements}

I thank all my collaborators for sharing their insight into the topics discussed here. 


\section{References}

[1] S. Weinberg, "Pion scattering lengths," Phys. Rev. Lett. 17 (1966) 616.

[2] J. Gasser and H. Leutwyler, "Low-Energy Theorems as Precision Tests of QCD," Phys. Lett. B 125 (1983) 325 .

[3] J. Bijnens, G. Colangelo, G. Ecker, J. Gasser and M. E. Sainio, "Elastic pi pi scattering to two loops," Phys. Lett. B 374 (1996) 210 [hep-ph/9511397].

[4] G. Colangelo, J. Gasser and H. Leutwyler, “The pi pi S wave scattering lengths,” Phys. Lett. B 488 (2000) 261 [hep-ph/0007112].

[5] H. Leutwyler, private communication.

[6] J. Gasser, B. Kubis and A. Rusetsky, "Cusps in K -> 3pi decays: a theoretical framework," Nucl. Phys. B 850 (2011) 96 [arXiv:1103.4273 [hep-ph]].

[7] J. R. Batley et al. [NA48-2 Collaboration], "Precise tests of low energy QCD from K(e4)decay properties,” Eur. Phys. J. C 70 (2010) 635.

[8] B. Adeva, et al., "Determination of $\pi \pi$ scattering lengths from measurement of $\pi^{+} \pi^{-}$atom lifetime," Phys. Lett. B 704 (2011) 24 [arXiv:1109.0569 [hep-ex]].

[9] P. A. Boyle et al., “K(13) semileptonic form-factor from 2+1 flavour lattice QCD,” Phys. Rev. Lett. 100 (2008) 141601 [arXiv:0710.5136 [hep-lat]].

[10] C. Allton et al. [RBC-UKQCD Collaboration], "Physical Results from 2+1 Flavor Domain Wall QCD and SU(2) Chiral Perturbation Theory,” Phys. Rev. D 78 (2008) 114509 [arXiv:0804.0473 [hep-lat]].

[11] V. Bernard, S. Descotes-Genon and G. Toucas, "Chiral dynamics with strange quarks in the light of recent lattice simulations,” JHEP 1101 (2011) 107 [arXiv:1009.5066 [hep-ph]].

[12] B. Moussallam, “N(f) dependence of the quark condensate from a chiral sum rule," Eur. Phys. J. C 14 (2000) 111 [hep-ph/9909292].

[13] H. Fukaya et al. [JLQCD and TWQCD Collaboration], "Determination of the chiral condensate from QCD Dirac spectrum on the lattice,” Phys. Rev. D 83 (2011) 074501 [arXiv:1012.4052 [hep-lat]].

[14] R. W. Griffith, “Scalar density terms, k-pi and k-k scattering lengths, and a symmetry-breaking parameter," Phys. Rev. 176 (1968) 1705.

[15] V. Bernard, N. Kaiser and U.-G. Meißner, “Threshold parameters of pi K scattering in QCD,” Phys. Rev. D 43 (1991) 2757.

[16] V. Bernard, N. Kaiser and U.-G. Meißner, "pi K scattering in chiral perturbation theory to one loop," Nucl. Phys. B 357 (1991) 129.

[17] J. Bijnens, P. Dhonte and P. Talavera, “pi K scattering in three flavor ChPT,” JHEP 0405 (2004) 036 [hep-ph/0404150].

[18] P. Buettiker, S. Descotes-Genon and B. Moussallam, "A new analysis of pi K scattering from Roy and Steiner type equations,” Eur. Phys. J. C 33 (2004) 409 [hep-ph/0310283].

[19] A. Poluektov et al. [Belle Collaboration], "Measurement of phi(3) with Dalitz plot analysis of B+$\longrightarrow$ D**(*) K+- decay,” Phys. Rev. D 70 (2004) 072003 [hep-ex/0406067].

[20] E. M. Aitala et al. [E791 Collaboration], "Model independent measurement of S-wave K- pi+ systems using D+ $\longrightarrow$ K pi pi decays from Fermilab E791," Phys. Rev. D 73 (2006) 032004 [Erratum-ibid. D 74 (2006) 059901] [hep-ex/0507099]. 
[21] J. M. Link et al. [FOCUS Collaboration], "The K- pi+ S-wave from the D+ -> K- pi+ pi+ Decay," Phys. Lett. B 681 (2009) 14 [arXiv:0905.4846 [hep-ex]].

[22] B. Aubert et al. [BABAR Collaboration], "Improved measurement of the CKM angle $\gamma$ in $B^{\mp} \rightarrow D^{(*)} K^{(* \mp)}$ decays with a Dalitz plot analysis of $D$ decays to $K_{S}^{0} \pi^{+} \pi^{-)}$and $K_{S}^{0} K^{+} K^{-)}$," Phys. Rev. D 78 (2008) 034023 [arXiv:0804.2089 [hep-ex]].

[23] C. B. Lang, L. Leskovec, D. Mohler and S. Prelovsek, "K pi scattering for isospin 1/2 and 3/2 in lattice QCD,” Phys. Rev. D 86 (2012) 054508 [arXiv:1207.3204 [hep-lat]].

[24] V. Bernard, N. Kaiser and U.-G. Meißner, "Chiral corrections to the S wave pion - nucleon scattering lengths,” Phys. Lett. B 309 (1993) 421 [hep-ph/9304275].

[25] V. Bernard, N. Kaiser and U.-G. Meißner, "Chiral prediction for the pi N S wave scattering length ato order O(M(pi)(4)),” Phys. Rev. C 52 (1995) 2185 [hep-ph/9506204].

[26] N. Fettes and U.-G. Meißner, "Pion nucleon scattering in chiral perturbation theory. 2.: Fourth order calculation,” Nucl. Phys. A 676 (2000) 311 [hep-ph/0002162].

[27] J. M. Alarcon, J. M. Camalich and J. A. Oller, "Improved description of the $\pi N$-scattering phenomenology in covariant baryon chiral perturbation theory," arXiv:1210.4450 [hep-ph].

[28] J. Gasser, V. E. Lyubovitskij and A. Rusetsky, "Hadronic atoms in QCD + QED," Phys. Rept. 456 (2008) 167 [arXiv:0711.3522 [hep-ph]].

[29] J. Gasser, M. A. Ivanov, E. Lipartia, M. Mojzis and A. Rusetsky, "Ground state energy of pionic hydrogen to one loop,” Eur. Phys. J. C 26 (2002) 13 [hep-ph/0206068].

[30] V. Baru, C. Hanhart, M. Hoferichter, B. Kubis, A. Nogga and D. R. Phillips, "Precision calculation of threshold $\pi^{-} d$ scattering, pi N scattering lengths, and the GMO sum rule,” Nucl. Phys. A 872 (2011) 69 [arXiv:1107.5509 [nucl-th]].

[31] D. Gotta, et al., “Pionic hydrogen,” Lect. Notes Phys. 745 (2008) 165.

[32] T. Strauch, et al., "Pionic deuterium,” Eur. Phys. J. A 47 (2011) 88 [arXiv:1011.2415 [nucl-ex]].

[33] R. H. Dalitz and S. F. Tuan, “A possible resonant state in pion-hyperon scattering,” Phys. Rev. Lett. 2 (1959) 425.

[34] R. H. Dalitz and S. F. Tuan, "The phenomenological description of -K -nucleon reaction processes," Annals Phys. 10 (1960) 307.

[35] U.-G. Meißner, U. Raha and A. Rusetsky, “Spectrum and decays of kaonic hydrogen," Eur. Phys. J. C 35 (2004) 349 [hep-ph/0402261].

[36] M. Bazzi, et al., “A New Measurement of Kaonic Hydrogen X rays,” Phys. Lett. B 704 (2011) 113 [arXiv:1105.3090 [nucl-ex]].

[37] Y. Ikeda, T. Hyodo and W. Weise, "Improved constraints on chiral SU(3) dynamics from kaonic hydrogen,” Phys. Lett. B 706 (2011) 63 [arXiv:1109.3005 [nucl-th]].

[38] Y. Ikeda, T. Hyodo and W. Weise, "Chiral SU(3) theory of antikaon-nucleon interactions with improved threshold constraints,” Nucl. Phys. A 881 (2012) 98 [arXiv:1201.6549 [nucl-th]].

[39] M. Mai and U.-G. Meißner, "New insights into antikaon-nucleon scattering and the structure of the Lambda(1405),” Nucl. Phys. A 900 (2013) 51 [arXiv:1202.2030 [nucl-th]].

[40] Z.-H. Guo and J. A. Oller, "Meson-baryon reactions with strangeness -1 within a chiral framework," Phys. Rev. C 87 (2013) 035202 [arXiv:1210.3485 [hep-ph]]. 
[41] A. Cieply and J. Smejkal, "Chirally motivated $\bar{K} N$ amplitudes for in-medium applications," Nucl. Phys. A 881 (2012) 115 [arXiv:1112.0917 [nucl-th]].

[42] B. Borasoy, U.-G. Meißner and R. Nißler, "K- p scattering length from scattering experiments," Phys. Rev. C 74 (2006) 055201, [hep-ph/0606108].

[43] S. Weinberg, "Evidence That the Deuteron Is Not an Elementary Particle," Phys. Rev. 137 (1965) B672.

[44] V. Baru, J. Haidenbauer, C. Hanhart, Y. Kalashnikova and A. E. Kudryavtsev, "Evidence that the $\mathrm{a}(0)(980)$ and $\mathrm{f}(0)(980)$ are not elementary particles," Phys. Lett. B 586 (2004) 53 [hep-ph/0308129].

[45] L. Liu, K. Orginos, F.-K. Guo, C. Hanhart and U.-G. Meißner, "Interactions of Charmed Mesons with Light Pseudoscalar Mesons from Lattice QCD and Implications on the Nature of the $D_{s 0}^{*}(2317)$," Phys. Rev. D 87 (2013) 014508 [arXiv:1208.4535 [hep-lat]].

[46] F.-K. Guo, C. Hanhart and U.-G. Meißner, "Interactions between heavy mesons and Goldstone bosons from chiral dynamics,” Eur. Phys. J. A 40 (2009) 171 [arXiv:0901.1597 [hep-ph]].

[47] Y.-R. Liu, X. Liu and S.-L. Zhu, "Light Pseudoscalar Meson and Heavy Meson Scattering Lengths," Phys. Rev. D 79 (2009) 094026 [arXiv:0904.1770 [hep-ph]].

[48] L. S. Geng, N. Kaiser, J. Martin-Camalich and W. Weise, "Low-energy interactions of Nambu-Goldstone bosons with $D$ mesons in covariant chiral perturbation theory," Phys. Rev. D 82 (2010) 054022 [arXiv:1008.0383 [hep-ph]].

[49] M. Cleven, F.-K. Guo, C. Hanhart and U.-G. Meißner, "Light meson mass dependence of the positive parity heavy-strange mesons," Eur. Phys. J. A 47 (2011) 19 [arXiv:1009.3804 [hep-ph]].

[50] P. Wang and X. G. Wang, "Study on $0^{+}$states with open charm in unitarized heavy meson chiral approach,” Phys. Rev. D 86 (2012) 014030 [arXiv:1204.5553 [hep-ph]].

[51] A. Faessler, T. Gutsche, V. E. Lyubovitskij and Y.-L. Ma, "Strong and radiative decays of the D(s0)*(2317) meson in the DK-molecule picture,” Phys. Rev. D 76 (2007) 014005 [arXiv:0705.0254 [hep-ph]].

[52] M. F. M. Lutz and M. Soyeur, "Radiative and isospin-violating decays of D(s)-mesons in the hadrogenesis conjecture,” Nucl. Phys. A 813 (2008) 14 [arXiv:0710.1545 [hep-ph]].

[53] F.-K. Guo, C. Hanhart, S. Krewald and U.-G. Meißner, "Subleading contributions to the width of the D*(s0)(2317),” Phys. Lett. B 666 (2008) 251 [arXiv:0806.3374 [hep-ph]].

[54] S. Godfrey, "Testing the nature of the $\mathrm{D}(\mathrm{sJ}) *(2317)+$ and $\mathrm{D}(\mathrm{sJ})(2463)+$ states using radiative transitions," Phys. Lett. B 568 (2003) 254 [hep-ph/0305122].

[55] B. Adeva, et al., "Evidence for pi K atoms with DIRAC," Phys. Lett. B 674 (2009) 11 [arXiv:0905.0101 [hep-ex]].

[56] J. Schacher, private communication.

[57] M. Döring and U.-G. Meißner, "Kaon-nucleon scattering lengths from kaonic deuterium experiments revisited,” Phys. Lett. B 704 (2011) 663 [arXiv:1108.5912 [nucl-th]].

[58] N. V. Shevchenko, "Near-threshold $K^{-} d$ scattering and properties of kaonic deuterium," Nucl. Phys. A 890-891 (2012) 50 [arXiv:1201.3173 [nucl-th]].

[59] M. Faber, M. P. Faifman, A. N. Ivanov, J. Marton, M. Pitschmann and N. I. Troitskaya, "Energy Level Displacement of Excited np State of Kaonic Deuterium In Faddeev Equation Approach,” Phys. Rev. C 84 (2011) 064314 [arXiv:1012.3933 [nucl-th]]. 